\title{
Substantiation of rational relationships of main parameters of the rock washing-out process when applying GD-300 hydraulic monitors at Kuzbass open pits
}

\author{
Daniil Poklonov ${ }^{1}$, Ilya Mironenko², Sergey Protasov², and Pavel Samusev ${ }^{2}$ \\ ${ }^{1}$ Kuzbass Design Company LLC, 650036, Kemerovo, 41, Tereshkova str, the Russian Federation \\ ${ }^{2}$ T.F. Gorbachev Kuzbass State Technical University, 650000 Kemerovo, 28, Vesennaya st., Russian \\ Federation
}

\begin{abstract}
The need to use hydraulic technology for washing out quaternary bedrock, as well as heterogeneous rocks previously piled in hydraulic-mine dumps located above commercial coal reserves, requires the use of modern high-performance equipment. As such equipment, it is advisable to use the new GD-300 hydraulic mining giants - monitors, which are designed, manufactured and tested at Kuzbass open pits. In order to increase the performance of hydraulic monitors at Kuzbass open pits, the following dependencies are determined in the article: changes in the amount of water flow through the hydraulic monitors on the nozzle diameter and the pressure in front of it; changes in the amount of solid rock washed out by the hydraulic monitor - solid flow on the nozzle diameter at various values of the pressure in front of it; changes in the solid flow on the pressure in front of the nozzle.
\end{abstract}

\section{Introduction}

At present, hydraulic dumps, in which pulp of quaternary overburden rock washed out by means of hydraulic mining from neighboring sections was previously piled, are an obstacle for mining a number of sections of commercial coal reserves in Kuzbass [1]. Considering that the mining in such rocks is difficult, especially in hydraulic-mine dump areas far from the pulp discharge point, a new hydraulic mining technology has been developed, including the use of hydraulic dredges for dredging water-bearing unconsolidated clay rocks, as well as water jet and hydraulic dredging units for washing out and dredging loamy rocks, as well as sandy-loamy sand after preliminary loosening them with a dragline excavator [2]. At the same time, a feature of the proposed technology is that part of these rocks is washed out with the combined use of a hydraulic dredge and a hydraulic monitor, when the processes of rock washing-out on the top bench and hydraulic dredging are implemented successively in the same production train [3-4].

To ensure conditions for the stable operation of such a hydraulic unit, it is required to determine and maintain certain washing-out parameters with fairly high accuracy $[5,6]$.

Moreover, the analysis shows that GD-300 hydraulic monitors manufactured by Gidromash LLC, experimental studies of which were carried out in 2013 at the Kedrovsky open pit [3-4] are able to provide high-performance water jetting of heterogeneous types of 
dump rocks. The GD-300 hydraulic monitor and its analogue T-521, manufactured by Yurginsky Machine-Building LLC and tested at the Taldinsky open-pit mine, have increased, in comparison with the widespread GDM-250M hydraulic monitor, flow-through sections of flow channels and nozzle diameters. Using nozzles with a diameter of $125 \div 175$ $\mathrm{mm}$, depending on the pressure in the water supply system, allows getting a water flow rate of up to $5000 \mathrm{~m}^{3} / \mathrm{h}$, i.e. two and a half times more than that of the GDM-250M hydraulic monitor [7]. New hydraulic monitors have already proven their advantages at Kuzbass two open pits; they are also used for mining water-bearing brown coal deposits of KATEK [8]. To ensure the efficient operation of such hydraulic monitors, substantiation of their rational parameters is required.

The physical and mechanical properties of rocks, which determine the intensity of their washing-out and destruction by a jet stream, are characterized by many parameters. Therefore, all rocks are divided into classes of difficulty in their mining by hydraulic means. This classification is based on the rock type and its particle size distribution, which indirectly take into account the physical and mechanical properties that affect the water jetting performance. In addition, an increase in the height of the mined bench leads to an increase in the solid flow (ceteris paribus) and, as a result, to a decrease in the specific water consumption. In this case, the volume of collapsing rock increases per unit length of the cut, and when the collapsed rock is washed out, the specific water consumption in most cases is significantly less than with cutting. On the other hand, when washing out high benches, the distance between the hydraulic monitor and the face, admissible under the safety condition, increases, decreasing its solid flow. The magnitude of the width of the drawn up and the hydraulic monitor advance increment are determined by the volume of rock mined from the same location of the hydraulic monitor, which also affects the hydraulic unit performance.

All of the above factors affect the efficiency of the washing-out process, therefore, to provide the procedures to calculate the rationale for using the new GD-300 (or T-521) hydraulic monitor when implementing a new hydraulic mining technology for washing out and moving rocks previously washed into a hydraulic-mine dump to a new location, in which rock washing-out and hydraulic dredging processes are implemented successively in the same production train, it is necessary to determine the following dependencies:

- changes in the amount of water flow $Q_{\mathrm{B}}$ through the hydraulic monitors on the nozzle diameter $d_{\mathrm{H}}$ and the pressure in front of it $H_{\mathrm{H}}$;

- changes in the solid flow $Q_{\mathrm{T}}$ on the nozzle diameter $d_{\mathrm{H}}$ at various values of the pressure in front of it $H_{\mathrm{H}}$;

- changes in the solid flow $Q_{\mathrm{T}}$ on the pressure in front of the nozzle $H_{\mathrm{H}}$.

The hydrodynamic characteristics of the jet in contact with the face, which depend on the rate of water flow from the nozzle, have the greatest influence on the performance of hydraulic monitors. At the same time, the quality of the jet is determined by its compactness, which, in turn, is estimated by such hydrodynamic parameters as jet diameter, axial dynamic pressure, jet impact force and average specific pressure on the face.

The quality of the jet stream is usually evaluated by its compactness. At the same time, the jet compactness and the parameters characterizing it depend primarily on the diameter of the nozzle and the pressure in front of it, as well as on the conditions of its formation in the flow channels of the hydraulic monitor and the nozzle (the presence or absence of dampers and other structural elements to eliminate circulation and vortex flows).

At the same time, the jet compactness deteriorates with increasing distance from the nozzle to the washed-out face, since the jet breaks up as a result of its interaction with the environment, resulting in an increase in its diameter. This leads to a decrease in the axial dynamic pressure, the total force of the jet impact and the average specific pressure. As a 
result of this, the rate of rock destruction with a jet stream decreases. Therefore, the identification of the dependences of the dynamic pressure of the jet on its initial parameters (nozzle diameter and pressure in front of it) and the distances between the nozzle of the hydraulic monitor and the face plane will allow, based on the required contact dynamic pressures, determining the necessary initial parameters of the jets and their influence on the hydraulic monitor performance.

The hydrodynamic properties of the hydraulic monitor jets can vary depending on the choice of the initial jet parameters. At the same time, some mining factors will be indirectly taken into account. Therefore, it is advisable to consider the influence of the hydrodynamic characteristics of the jet on the hydraulic monitor efficiency.

The main hydrodynamic parameter of the hydraulic monitor jet, which determines its compactness and washing-out capacity, is the axial dynamic pressure $p_{m}$. Experimental studies [9] found that the axial dynamic pressure is associated with the length of the initial section of the jet by the following relation:

$$
\frac{p_{m}}{p_{0}}=\left(\frac{l_{i}}{l}\right)^{k},
$$

Where $p_{0}$ is the water pressure in front of the nozzle, $\mathrm{Pa} ; l_{i}$ is the length of the initial section of the jet, within which there is a core of a continuous unbroken stream that moves at a constant rate equal to the rate of flow out of the nozzle, $l$ is the distance from the nozzle to the face plane at some point in time, $\mathrm{m} ; k$ is an exponent characterizing the rate of the hydraulic monitor jet break-up.

Experimental studies [8] found that for low-pressure jets (up to $2 \mathrm{MPa}$ ), which are mainly used in open pit mining, and nozzles with a diameter of 50 to $190 \mathrm{~mm}$, the exponent is $k=0,5$. From equation (1) we obtain

$$
p_{m}=p_{0}\left(\frac{l_{i}}{l}\right)^{0,5}
$$

The initial section is characterized by the water jet compactness: the longer its length, the more compact is the jet and the greater is the axial dynamic pressure at equal distances from the nozzle. The length of the initial section depends on the Reynolds number at the jet flow out from the nozzle and on its diameter [10]. For low-pressure hydraulic monitor jets used in open cast mining, this dependence is described by the equation [9]

$$
\frac{l_{i}}{d_{i}}=A-B \cdot R_{\mathrm{e}},
$$

where $d_{\mathrm{H}}$ - the nozzle diameter, $\mathrm{m} ; A$ and $B$ - empirical coefficients; $R_{\mathrm{e}}-$ Reynolds number for the jet at nozzle exit

$$
R_{\mathrm{e}}=\frac{v_{0} d_{i}}{v}
$$

where $v_{0}-$ nozzle flow rate, $\mathrm{m}$

$$
v_{0}=\varphi \sqrt{2 g H_{i}}
$$

$v=1,01 \cdot 10^{-6} \mathrm{~m}^{2} / \mathrm{s}-$ kinematic viscosity coefficient at water temperature $t=20^{\circ} \mathrm{C}$; $\varphi=0,92-0,96-$ rate coefficient; $g$ - acceleration of gravity, $\mathrm{m} / \mathrm{s}^{2} ; H_{\mathrm{H}}-$ working water pressure in front of the nozzle, $m$.

Solving equation (3) with respect to $l_{\mathrm{H}}$, we obtain

$$
l_{i}=d_{i}\left(A-B \cdot R_{\mathrm{e}}\right)
$$

In view of (6), expression (2) is transformed to the form 


$$
p_{m}=p_{0} \sqrt{\frac{d_{i}\left(A-B \cdot R_{\mathrm{e}}\right)}{l}} .
$$

In turn

$$
p_{0}=\rho_{0} \frac{v_{0}^{2}}{2},
$$

where $\rho_{0}$ - water density, $\mathrm{kg} / \mathrm{m}^{3}$.

In view of (5), we obtain

$$
p_{0}=\rho_{0} \cdot \varphi^{2} \cdot g \cdot H_{i}
$$

Thus, the expression for determining the axial dynamic pressure depending on the distance from the nozzle to the plane of contact with the face will have the form

$$
p_{m}=\rho_{0} \cdot \varphi^{2} \cdot g \cdot H_{i} \sqrt{\frac{d_{i}\left(A-B \cdot R_{\mathrm{e}}\right)}{l}} .
$$

Analyze the dependence of the axial dynamic pressure on the initial parameters of the hydraulic monitor jet $\left(d_{\mathrm{H}}, H_{\mathrm{H}}\right)$ for the average distance $l_{\mathrm{cp}}(\mathrm{m})$ from the nozzle to the face during the period between two advance increments of the hydraulic monitor. This distance can be determined by the formula

$$
l_{\mathrm{cp}}=0,5 \cdot S+\varepsilon \cdot H_{\mathrm{y}}
$$

where $S$ is the optimal advance increments the hydraulic monitor, $\mathrm{m} ; \varepsilon=0,4$ - coefficient of proximity of the monitor to the face; $H_{\mathrm{y}}$ - the height of the bench being mined, $\mathrm{m}$.

In accordance with Typical technological schemes for mining at open pits (Moscow: Nedra, 1982), a height of the mined bench being $20 \mathrm{~m}$; the optimum advance increment for the hydraulic monitor is $18 \mathrm{~m}$. In view of it, the average distance will be $17 \mathrm{~m}$.

The results of the calculation of axial dynamic pressures of the hydraulic monitor jets depending on the pressure in front of the nozzle and its diameter are shown in Fig. 1.

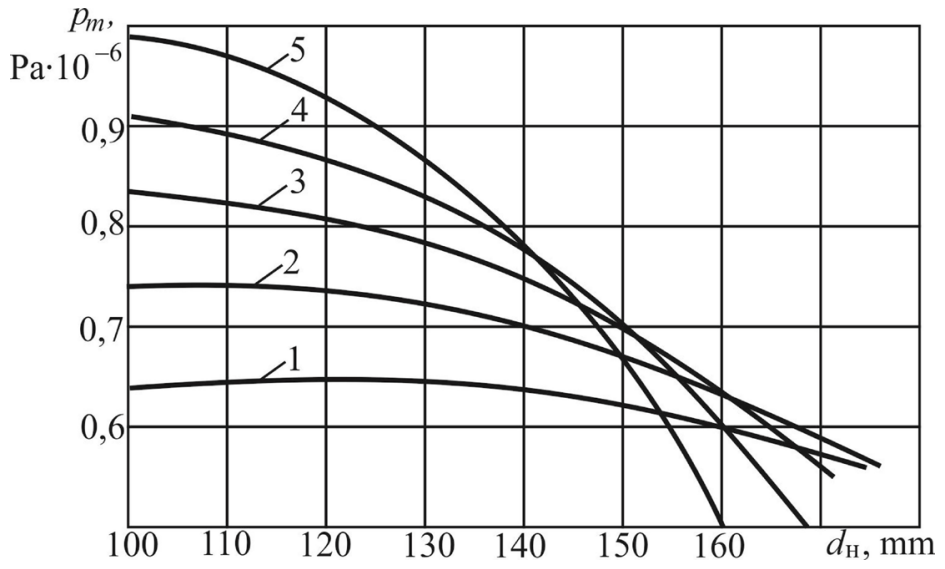

Fig. 1. Graphs of the dependence of axial dynamic pressures $P_{m}\left(\mathrm{~Pa} \cdot 10^{-6}\right)$ in hydraulic monitor jets on nozzle diameter $(\mathrm{mm})$ and pressure in front of it $H_{H}(\mathrm{~m}): 1-100 \mathrm{~m}, 2-120 \mathrm{~m}, 3-140 \mathrm{~m}, 4-160 \mathrm{~m}$, $5-180 \mathrm{~m}$.

It can be seen from Fig. 1 that, in the studied limits, at all pressures in front of the nozzle of more than $120 \mathrm{~m}$, the axial dynamic pressure of the jet constantly decreases with increasing nozzle diameter. Moreover, the higher the pressure in front of the hydraulic monitor nozzle, the steeper is a curve of the axial dynamic pressure descending. Therefore, 
we can assume that for each nozzle diameter there is a head at which the axial dynamic pressure will be maximum.

To verify this, we plotted the dependence of the axial dynamic pressures of the jet stream on the pressure in front of the nozzle (Fig. 2). The numbers on the graphs indicate the pressure in front of the nozzle $H_{0}$, $\mathrm{m}$ for its various diameters.

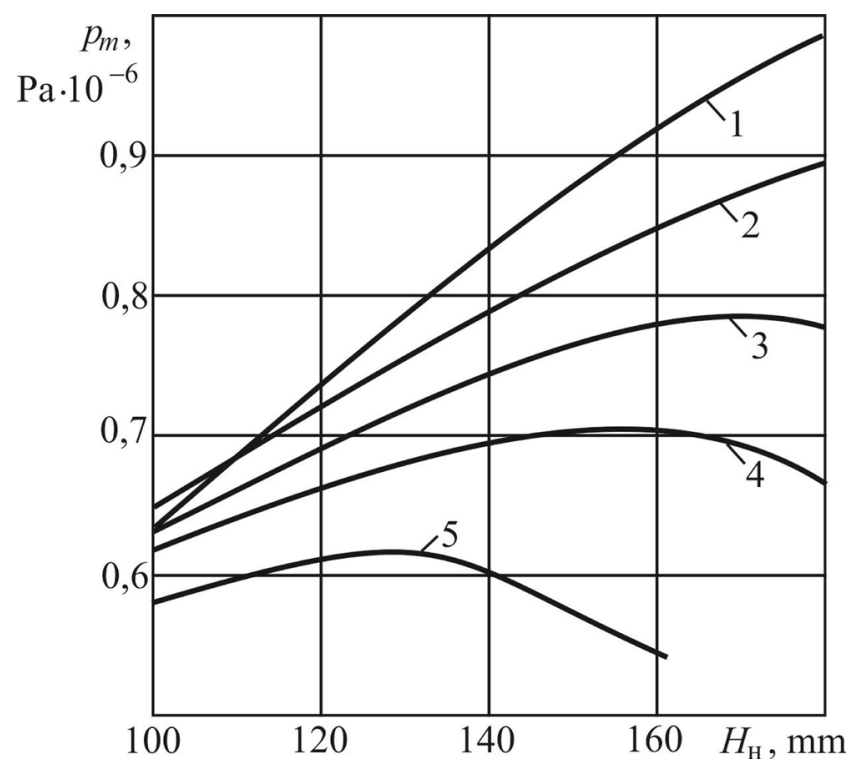

Fig. 2. Graphs of the dependence of axial dynamic pressures pm $(\mathrm{Pa} \cdot 10-6)$ in hydraulic monitor jets on pressure $\mathrm{HH}(\mathrm{m})$ in front of the nozzle and its diameter $\mathrm{dH}(\mathrm{mm}): 1-100 \mathrm{~mm}, 2-125 \mathrm{~mm}, 3-$ $140 \mathrm{~mm}, 4-150 \mathrm{~mm}, 5-165 \mathrm{~mm}$.

As the graphs show, for each nozzle diameter there is a head at which the axial dynamic pressure is maximum. These optimal pressures for the studied nozzles are: for nozzles with a diameter of $165 \mathrm{~mm}-130 \mathrm{~m}$; for nozzles with a diameter of $150 \mathrm{~mm}-160 \mathrm{~m}$ and for nozzles with a diameter of $140 \mathrm{~mm}-170 \mathrm{~m}$. For nozzles with a diameter of 100, 110 and $125 \mathrm{~mm}$, the optimal axial dynamic pressures are outside the field of pressure data. Therefore, for these nozzles it is advisable to apply the maximum pressure possible under the operating conditions.

As for the optimum diameters of nozzles at pressures of 100-120 m (see Fig. 2), it should be noted that such pressures are practically not used in modern production, therefore, this fact has no practical value.

The value of the axial dynamic pressure of the jet in contact with the face, ceteris paribus, completely determines the efficiency of rock washing-out by hydraulic monitors, which is primarily characterized by the specific washing-out water flow rate. V.F. Khnykin [11], having mathematically processed the results of experimental data, has established that the specific water consumption for water jetting, ceteris paribus, is inversely proportional to the value of axial dynamic pressure raised to the power of 0.5 , that is

$$
\frac{q_{1}}{q_{2}}=\frac{p_{m_{2}}^{0,5}}{p_{m_{1}}^{0,5}}
$$

where $q_{1}$ and $q_{2}$ - specific water consumption for water jetting with axial dynamic pressure in contact with the face $p_{m_{1}}$ and $p_{m_{2}}$, respectively. 
If in specific mining, geological and engineering conditions the specific water flow rate $q\left(\mathrm{~m}^{3} / \mathrm{m}^{3}\right)$ is known for a certain value of the nozzle diameter $d_{\mathrm{H}}$ and axial dynamic pressure $p_{m}(\mathrm{~Pa})$, then according to relation (12), the specific water flow rate $q_{i}$ in the same mining, geological and engineering conditions at axial dynamic pressure $p_{m_{1}}$ can be determined by the formula

$$
q_{1}=q \sqrt{\frac{p_{m}}{p_{m_{1}}}} .
$$

The experimental studies, on the basis of which the relation (12) was obtained, were carried out in production when mining a placer deposit including clay rocks of 111 mining difficulty class according to the unified classification of placer deposits [12]. Moreover, during the experiment, nozzles with a diameter of 50 to $90 \mathrm{~mm}$, heads from 30 to $100 \mathrm{~m}$ and distances from the nozzle to the face from 4 to $20 \mathrm{~m}$ were used.

At present, more powerful hydraulic monitors with nozzles with a diameter of 100-175 $\mathrm{mm}$ and heads from 100 to 200 are used. The results of the above experiments can be approximated to such hydraulic monitors and heads using relation (13) for rocks close in physical and mechanical properties to III class according to the indicated above unified classification of placer deposits.

Other classifications of rocks according to the difficulty of their washing-out by hydraulic monitors are used at open pits [9]. Rocks of the fifth mining difficulty class according to the classification used for coal mines are the closest in their physical and mechanical properties to soils of III mining difficulty class according to the unified classification of placer deposits. Nozzles with a diameter of $110 \mathrm{~mm}$ and a pressure in front of the nozzle of $120 \mathrm{~m}$ being used or these rocks; the specific flow rate for washing-out is $8.5 \mathrm{~m}^{3} / \mathrm{m}^{3}$. Based on these conditions, we will calculate the dependence of the specific water flow on the axial dynamic pressure according to relation (13). The calculation results are shown in Fig. 3.

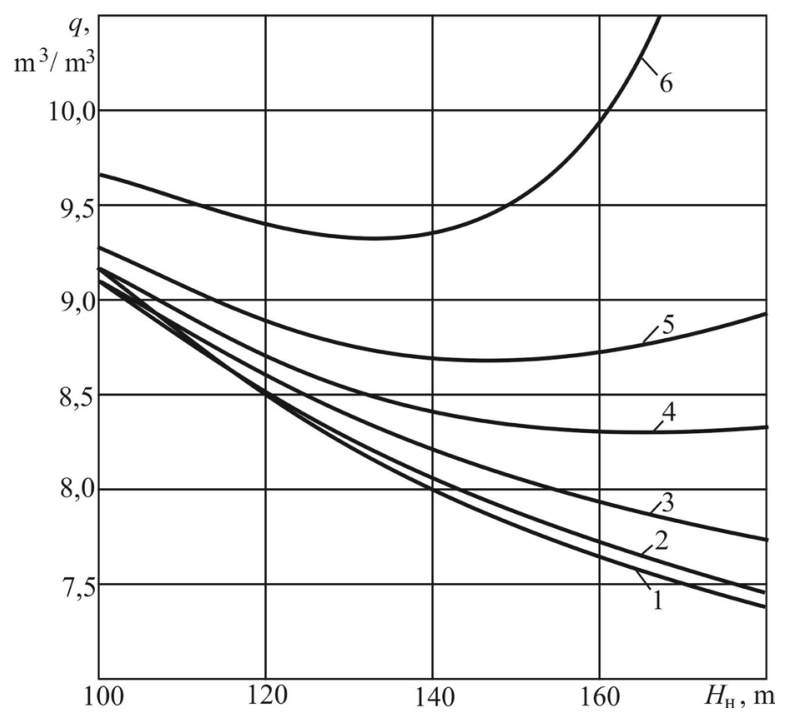

Fig. 3. Graphs of the dependence of the specific flow rate of water for water jetting on the pressure $H h(\mathrm{~m})$ in front of the nozzle and its diameter $d H(\mathrm{~mm}): 1-100 \mathrm{~mm}, 2-110 \mathrm{~mm}, 3-125 \mathrm{~mm}, 4-$ $140 \mathrm{~m}, 5-160 \mathrm{~mm}, 6-165 \mathrm{~mm}$. 
As can be seen from the graphs in Fig. 3, for each nozzle diameter there is a pressure head at which the specific water consumption is minimal. Moreover, these minima correspond to the maxima of the axial dynamic pressure in contact with the face (see Fig. 2 ). The specific flow rate of water for washing-out allows determining the solid flow at a known water flow rate. The water flow through the hydraulic monitor $Q_{\mathbf{B}}\left(\mathrm{m}^{3} / \mathrm{h}\right)$ is determined by the well-known formula [12]

$$
Q_{\mathrm{B}}=3600 \mu \omega \sqrt{2 g H_{\mathrm{H}}},
$$

where $\mu=\alpha \varphi$ - consumption rate; $\alpha=1$ - jet compression ratio; $\omega$ - cross-sectional area of the nozzle, $\mathrm{m}^{2}$.

Since $\omega=\pi d_{\mathrm{H}}^{2} / 4$, then formula (14) can be transformed to the form

$$
Q_{\mathrm{B}}=11765 d_{\mathrm{H}}^{2} \sqrt{H_{\mathrm{H}}} \text {. }
$$

The results of calculating the water flow through the hydraulic monitor according to the formula (15) are shown in Fig. 4.

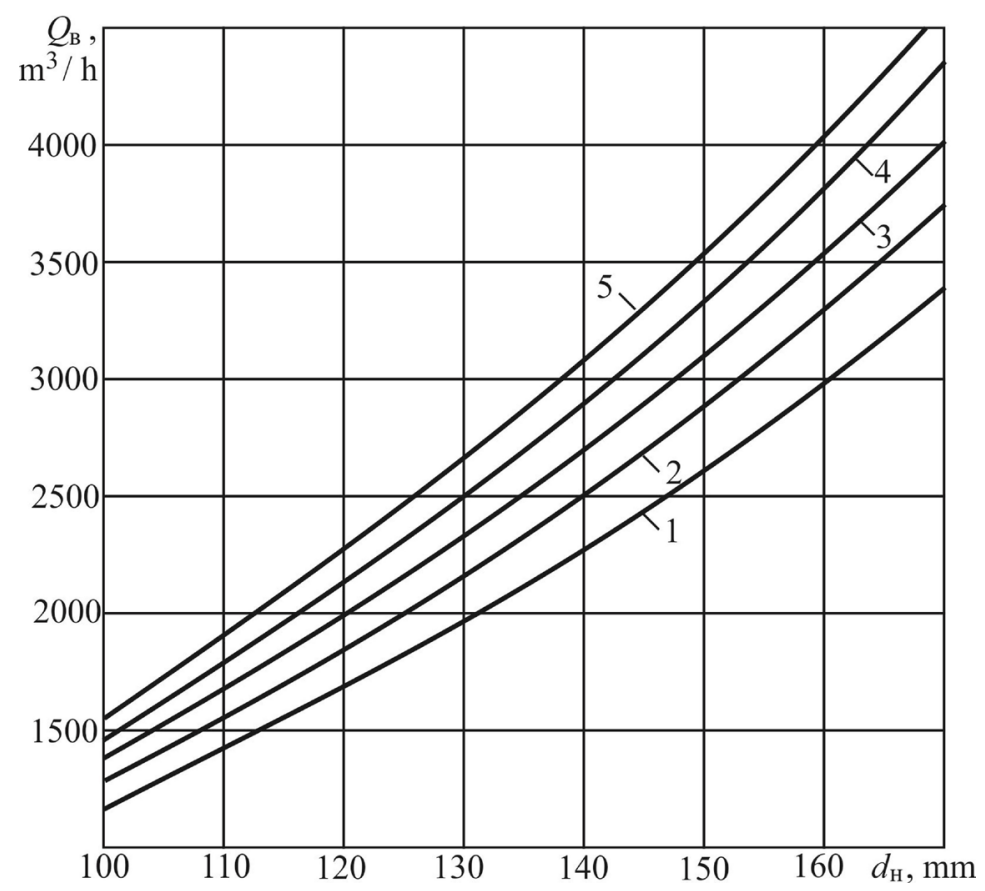

Fig. 4. Graphs of the dependence of the rate of water flow through the hydraulic monitor on the nozzle diameter $d H$ and pressure in front of it: $H h: 1-100 \mathrm{~m}, 2-120 \mathrm{~m}, 3-140 \mathrm{~m}, 4-160 \mathrm{~m}, 5-$ $180 \mathrm{~m}$.

The data obtained allow calculating the solid flow at different values of the diameter of the nozzle and the pressure in front of it, using the formula

$$
Q_{\mathrm{T}}=\frac{Q_{\mathrm{B}}}{q} \text {. }
$$

The results of calculating the solid flow by the formula (16) are shown in Figures 5 and 6 


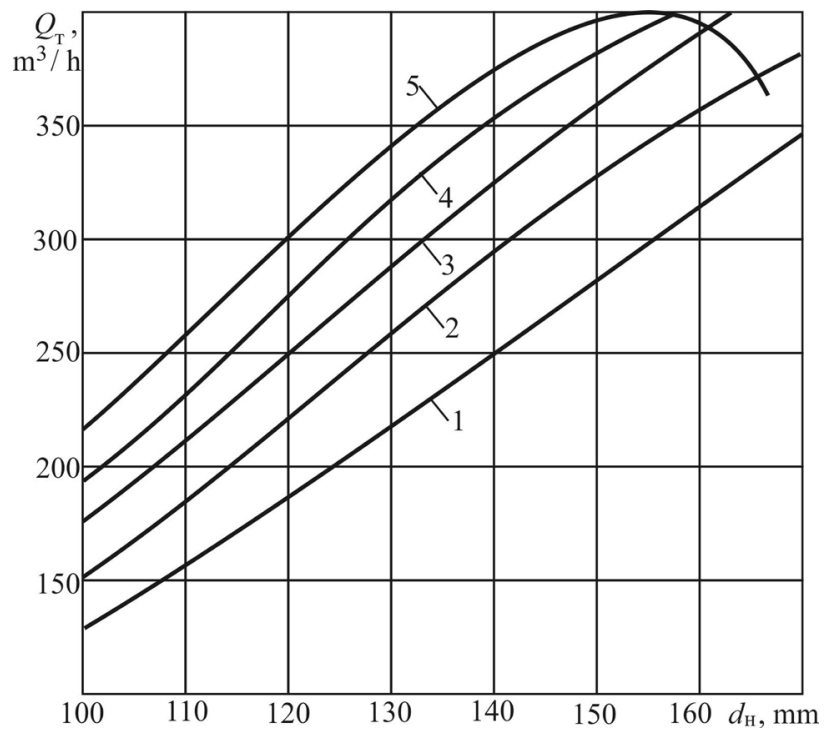

Fig. 5. Graphs of the dependence of the solid flow $Q_{\mathrm{T}}$ on the nozzle diameter $d H$ at various pressure values in front of it $H h: 1-100 \mathrm{~m}, 2-120 \mathrm{~m}, 3-140 \mathrm{~m}, 4-160 \mathrm{~m}, 5-180 \mathrm{~m}$.

For nozzles with a diameter of $165 \mathrm{~mm}$, as the graphs in Fig. 6 show, the solid flow corresponds to a head $H_{\mathrm{H}}=155 \mathrm{~m}$. Therefore, for pressures of more than $150 \mathrm{~m}$, nozzles with a diameter of $165 \mathrm{~mm}$ are inefficient to use, which is also confirmed by the graphs of the specific rate of water flow for jetting on the nozzle diameter and pressure in front of it (see Fig. 3).

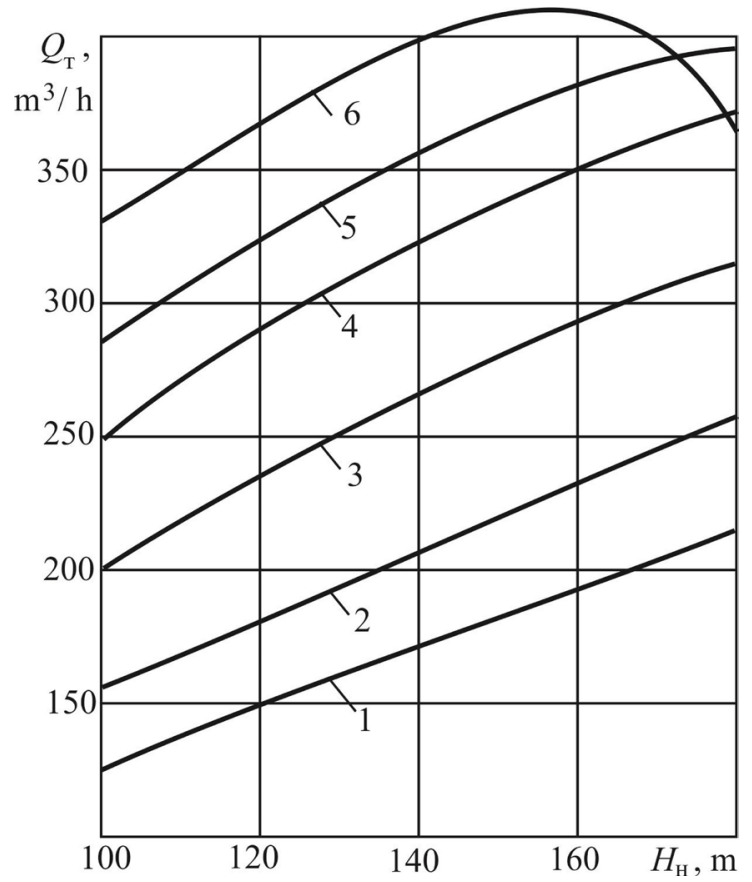

Fig. 6. Graphs of the dependence of the solid flow Qt on the pressure in front of the nozzle $H h$ : 1 $100 \mathrm{~mm}, 2-110 \mathrm{~mm}, 3-125 \mathrm{~mm}, 4-140 \mathrm{~mm}, 5-150 \mathrm{~mm}, 6-165 \mathrm{~mm}$. 
In addition, it should be noted that with an increase in the nozzle diameter from 100 to $150 \mathrm{~mm}$, the water flow through the hydraulic monitor increases 2.25 times, while the solid flow increases only 1.8-2.2 times. This is explained by a decrease in the specific water consumption for water jetting with an increase in the nozzle diameter. Therefore, when choosing a hydraulic monitor, this fact must be taken into account.

Based on the results of the above studies, it can be argued that when mining $\mathrm{V}$ mining difficulty class rocks, which are the bulk of the rocks mined by the hydraulic method at the Kuzbass open pits, the GD-300 (T-521) hydraulic monitors solid flow rate varies in the range from $300-320 \mathrm{~m}^{3} / \mathrm{h}$ to $380-400 \mathrm{~m}^{3} / \mathrm{h}$. It should be taken into consideration that for each nozzle there is a certain pressure at which the specific flow rate will be minimal. This factor determines the rational ratio of the main parameters of water jetting when using GD300 (T-521) hydraulic monitors and, which makes it possible to determine the solid flow range for each nozzle, in which it is expedient to use it. Rational ratios of the main parameters of the rock washing-out process and the specific consumption of water when using GD-300 (T-521) hydraulic monitors are presented in Table 1.

Table 1. Rational ratios of solid flow, water pressure in front of the nozzle, nozzle diameter and specific water consumption when using GD-300 (T-521) hydraulic monitors.

\begin{tabular}{|c|c|c|c|}
\hline $\begin{array}{c}\text { Solid flow } \\
Q_{\mathrm{T}}, \mathbf{m}^{\mathbf{3}} \mathbf{h}\end{array}$ & $\begin{array}{c}\text { Pressure in front of } \\
\text { the nozzle } H_{\mathbf{H}}, \mathbf{m}\end{array}$ & $\begin{array}{c}\text { Nozzle diameter } \\
d_{0}, \mathbf{m m}\end{array}$ & $\begin{array}{c}\text { Specific water } \\
\text { consumption } q, \mathbf{~ m}^{\mathbf{3}} / \mathbf{m}^{\mathbf{3}}\end{array}$ \\
\hline $320-340$ & $180-200$ & 125 & $7,3-7,0$ \\
\hline $350-370$ & $160-180$ & 140 & 8,3 \\
\hline $360-380$ & $140-160$ & 150 & 8,7 \\
\hline $370-400$ & $120-140$ & 165 & 9,4 \\
\hline
\end{tabular}

Thus, the required solid flow being known; the necessary approximate pressure in front of the nozzle and its diameter can be determined according to Table 1, as well as the specific water flow rate that GD-300 (or T-521) hydraulic monitors can provide. These data allow further selection of pumping equipment for the water supply system for water jetting.

\section{References}

1. I.A. Mironenko, S.I. Protasov, Journal of Mining and Geotechnical Engineering, 1, 24 (2019)

2. RF patent 2661950. The Method of hydraulic-mine dump re-piling. Bul. \#21 (2018)

3. RF patent 2681772. The Method of hydraulic rock re-piling. Bul. \#8 (2019)

4. RF patent 2691252. The Method of re-piling hydraulic-mine dump rock with hydraulic monitor and hydraulic dredger. Bul. \#17 (2019)

5. S.I. Protasov, I.A. Mironenko, Mine surveying and subsoil use, 3, 35 (2019)

6. S.I. Protasov, I.A. Mironenko, Mining informational and analytical bulletin (scientific and technical journal), 10, 55 (2019)

7. S.I. Protasov, D.A. Poklonov, Mining informational and analytical bulletin (scientific and technical journal), 5, 115 (2016)

8. A.V. Reznik, V.I. Cheskidov, Journal of mining science, 1, 106 (2019)

9. S.S. Shavlovskiy, The basics of the jet dynamics when collapsing of the massif (Nauka, Moscow, 1979)

10. I.S. Kuklin, K.M. Shtukaturov, Research papers of Institute of Mining of Ural Departament of USSR Academy of Science, 3, 12 (1963) 
11. V.F. Khnykin, Destruction of rocks by hydraulic monitor jets in open cast mining (Nauka, Moscow, 1969)

12. G.A. Nurok, Processes and technology for hydraulic mechanization of open cast mining (Nedra, Moscow, 1985) 